\title{
Quantum Entanglement in Heisenberg Antiferromagnets
}

\author{
V. Subrahmanyam \\ Department of Physics, Indian Institute of Technology, Kanpur, India.
}

\begin{abstract}
Entanglement sharing among pairs of spins in Heisenberg antiferromagnets is investigated using the concurrence measure. For a nondegenerate $\mathrm{S}=0$ ground state, a simple formula relates the concurrence to the diagonal correlation function. The concurrence length is seen to be extremely short. Various finite clusters are studied numerically, to see the trend in two dimensions. It is argued that the concurrences vanish between pairs of spins which are not nearest neighbors, for the linear chain and square lattice antiferromagnet ground states. For the triangular lattice and Kagome' lattice, nearest-neighbor concurrences also vanish. The concurrences in the maximal-spin states are explicitly calculated, where the concurrence averaged over all pairs is larger than the $\mathrm{S}=0$ states.
\end{abstract}

\section{INTRODUCTION}

In recent years quantum entanglement has emerged as a common platform for scientists working in various fields such computer science, physics, mathematics and chemistry [1]. In particular, quantum entanglement of spin- $\frac{1}{2}$ degrees of freedom, qubits, has been studied extensively, due to their importance for quantum computers, not to mention their well-known applicability in various condensed-matter systems, optics and other branches of physics. For a pure state of many qubits, quantum entanglement, which is quantified by the von Neumann entropy of the reduced density matrix, is a measure of how a subsystem is correlated to the rest of the system. The key ingredient for entanglement is the superposition of states. A linear combination of two pure states, both of which are entangled, viz. having a non-zero entropy for a subsystem, can exhibit no entropy at all. And the reverse situation is also possible, two pure states with no entanglement, can give rise to entanglement on superposition.

For a system consisting of a large number of qubits, how different pairs share entanglement in a pure state cannot easily be specified, even with known diagonal and off-diagonal correlation functions. We will show below, for the ground state Heisenberg antiferromagnet with zero total spin, the concurrence measure can be specified in terms of the diagonal correlation function alone. In a pure state of many qubits, a subsystem of two qubits, in general, will be in a mixed state. A mixed state density matrix can be written as a decomposition over pure state density matrices, with a large number of possible decompositions over pure states. The entanglement of the pair of qubits is the average entanglement of a decomposition, minimized over all possible decompositions. Starting from a given many-qubit state, with a density matrix $\rho=|\psi><\psi|$, the reduced density matrix $R_{i j}$ for a pair of qubits is constructed by performing a partial trace over the rest of the qubits to be eliminated, $R_{i j}=\operatorname{tr} \rho$. In general the reduced density matrix represents a mixed state for the pair of sites labeled $(i, j)$. The von Neumann entropy calculated from the eigenvalues $r_{n}$ of $R_{i j}$, as $-\sum r_{n} \log _{2} r_{n}$ quantifies entanglement of this pair with the rest of the qubits. The concurrence measure 2] has the important information as to how these qubits are entangled among themselves, and is given as

$$
C_{i j}=\max \left(0, \lambda_{1}^{1 / 2}-\lambda_{2}^{1 / 2}-\lambda_{3}^{1 / 2}-\lambda_{4}^{1 / 2}\right) .
$$

In the above $\lambda_{i}$ are the eigenvalues in decreasing order of the matrix $R \hat{R}$, where $R$ is the time-reversed matrix, $\hat{R}=\sigma_{y} \times \sigma_{y} R^{*} \sigma_{y} \times \sigma_{y}$.

The two-site concurrence depends only on the structure of entanglement of a many-qubit state without reference to a Hamiltonian. However, we would like to investigate the pairwise entanglement in the ground state of the Heisenberg Hamiltonian. We will study the entanglement in the ground state Heisenberg antiferromagnet, with a Hamiltonian

$$
H=\sum_{<i, j>} \frac{1}{2}\left(S_{i}^{+} S_{j}^{-}+S_{i}^{-} S_{j}^{+}\right)+S_{i}^{z} S_{j}^{z},
$$

The sum is over all nearest-neighbor pairs on a given lattice. Working in a diagonal basis of $S_{i}^{z}$ for every site $i$, there are two states per site, viz., $\uparrow$, and $\downarrow$. A manyspin state can be characterized by the number of down spins, as the total z-component of the spin, $S^{z}=\sum S_{i}^{z}$, is a good quantum number, in addition to the total spin quantum number $S$. The above Hamiltonian is also invariant under time reversal. The ground state will belong to $S^{z}=0$ subspace, as this sector has a representation state from every spin sector labeled by $S=0,1, . . N / 2$. For most lattices, and finite clusters the ground state belongs to $S=0$, for example the linear chain, the square lattice, the triangular lattice, Kagome' lattice, and finite clusters that will be considered below. However, the ground state energy and the wave function are known only for the case of the linear chain through the Bethe Anzatz [3] . The highest-energy state has $S=N / 2$, which is the maximum value for the total spin, with a degeneracy of $N+1$ corresponding to the different values of $S^{z}=-N / 2, . . N / 2$. This maximal-spin state would be the ground state, if the interaction is ferromagnetic in the above Hamiltonian (by changing the overall sign of the Hamiltonian). 


\section{CONCURRENCES IN $S=0$ STATES}

Let us consider a state with $\mathrm{m}$ down spins

$$
\left|\psi>=\sum \psi\left(x_{1} . . x_{m}\right)\right| x_{1} \ldots x_{m}>
$$

where the ordered labels $x_{1} . . x_{m}$ denote the locations of the down spins. Let us rewrite the state as

$$
\left|\psi>=\sum_{s_{1} . . s_{N}} \psi\left(s_{1} . . s_{N}\right)\right| s_{1} . . s_{N}>
$$

where $s_{i}$ is the eigenvalue of $S_{i}^{z}$ for the i'th qubit. The reduced density matrix $R_{i j}$ of two sites $i$ and $j(j>i)$ has the matrix elements

$$
R_{s_{i}, s_{j}}^{s_{i}^{\prime}, s_{j}^{\prime}}=\sum_{s_{1} . . s_{n}} \psi^{\star}\left(s_{1}, . . s_{i}^{\prime} . . s_{j}^{\prime} . . s_{n}\right) \psi\left(s_{1} . . s_{i} . . s_{j} . . s_{n}\right)
$$

where the sum is over all spin variables except at sites $i$ and $j$. Since the many-qubit state has a definite eigenvalue for $S^{z}$, we have $\left[R_{i j}, S_{i}^{z}+S_{j}^{z}\right]=0$. This in turn implies the following structure for the reduced density matrix

$$
R_{i j}=\left(\begin{array}{cccc}
v_{i j} & 0 & 0 & 0 \\
0 & w_{1 i j} & z_{i j}^{*} & 0 \\
0 & z_{i j} & w_{2 i j} & 0 \\
0 & 0 & 0 & u_{i j}
\end{array}\right)
$$

In the above we used the two-qubit basis states $\mid \uparrow \uparrow>$ ,$|\uparrow \downarrow>,| \downarrow \uparrow>, \mid \downarrow \downarrow>$, where $|\uparrow>,| \downarrow>$ stand for eigenstates of $S_{i}^{z}$ with eigenvalues $1 / 2,-1 / 2$ respectively.

The matrix elements can be expressed in terms of the expectation values, $\langle A>\equiv<\psi|A| \psi\rangle$, as

$$
u_{i, j}=<\left(\frac{1}{2}-S_{i}^{z}\right)\left(\frac{1}{2}-S_{j}^{z}\right)>, v_{i, j}=<\left(\frac{1}{2}+S_{i}^{z}\right)\left(\frac{1}{2}+S_{j}^{z}\right)>,
$$

$$
z_{i, j}=<S_{j}^{+} S_{i}^{-}>
$$

$w_{1 i j}=<\left(\frac{1}{2}+S_{i}^{z}\right)\left(\frac{1}{2}-S_{j}^{z}\right)>, w_{2 i j}=<\left(\frac{1}{2}-S_{i}^{z}\right)\left(\frac{1}{2}+S_{j}^{z}\right)>$

The diagonal matrix elements are simply related to the diagonal correlation functions, and the off-diagonal matrix element is just the off-diagonal correlation function. The concurrence for the two sites has now a simpler form

$$
C_{i j}=2 \max \left(0,\left|z_{i j}\right|-\sqrt{u_{i j} v_{i j}}\right)
$$

As can easily be seen from above, the concurrence measure uses both diagonal and off-diagonal correlation functions. Whether or not two sites have a non-zero concurrence is not at all intuitive, given a specific state with known correlation functions. For a long-ranged concurrence, the necessary condition is the existence of off-diagonal long range order (ODLRO). If there is no off-diagonal long range order (ODLRO), $z_{i j} \rightarrow 0$, as $\left|\vec{r}_{i}-\vec{r}_{j}\right| \rightarrow \infty$, the concurrence of two sites far apart would go to zero. Thus, the existence of ODLRO is a necessary condition for a long-ranged concurrence. However, even with a ODLRO, long-ranged concurrence may be absent, if $\left|z_{i j}\right|<\sqrt{u_{i j} v_{i j}}$. We will see below that the pairwise concurrence is short ranged in the ground state of Heisenberg spin systems. For a system with a large number of qubits, only the nearest-neighbor concurrence seems to be nonzero.

Let us consider a nondegenerate $S=0$ state, which can be represented in $S^{z}=0$ subspace. Because of the timereversal symmetry of the Hamiltonian, the wave function can be chosen to be real. This would imply that the off-diagonal matrix element $z_{i j}$ of the reduced density matrix will be real. Further, the rotational symmetry of the Hamiltonian would imply, for the $S=0$ sector, $<S_{i}^{x} S_{j}^{x}>=<S_{i}^{y} S_{j}^{y}>=<S_{i}^{z} S_{j}^{z}>$. Exploiting this property and denoting the diagonal correlation function by $\Gamma_{i j}=<S_{i}^{z} S_{j}^{z}>$, we have

$$
z_{i j}=<S_{j}^{+} S_{i}^{-}>=2 \Gamma_{i j}
$$

The time-reversal invariance also implies, $\left\langle S_{i}^{z}>=0\right.$, in this state. This will simplify the diagonal matrix elements of $R_{i j}$. Thus the diagonal elements of the reduced density matrix are

$$
v_{i j}=u_{i j}=\frac{1}{4}+\Gamma_{i j} .
$$

In addition the diagonal correlation function has the property $\left|\Gamma_{i j}\right| \leq 1 / 4$, since we are dealing with spin-1/2 species. Now the concurrence can be specified completely in terms of the diagonal correlation function alone.

The diagonal correlation function between two arbitrary spins can take both positive or negative values. For a pair of qubits with $\Gamma_{i j}>0, C_{i j}=2 \max \left(0,2 \Gamma_{i j}-\right.$ $\left.\frac{1}{4}-\Gamma_{i j}\right)=0$. The above argument would imply that the concurrence is zero for a pair of qubits, if their diagonal correlation function is positive, in a nondegenerate $S=0$ state. On a bipartite lattice, if two spins belong to the same sublattice, then the diagonal correlation function would be positive, implying the concurrence here is zero. On the other hand, when $\Gamma_{i j}<0$, then we have $\sqrt{u_{i j} v_{i j}}=1 / 4-\left|\Gamma_{i j}\right|$, so that the concurrence is given by $C_{i j}=2 \max \left(0,3\left|\Gamma_{i j}\right|-\frac{1}{4}\right)=6\left(\left|\Gamma_{i j}\right|-\frac{1}{12}\right)$ if positive, and otherwise zero. This gives us a simple test for a nonzero concurrence, viz. if $\Gamma_{i j}<0,\left|\Gamma_{i j}\right|>1 / 12$. Thus in a nondegenerate $S=0$ state, the concurrence for a pair of spins is specified entirely by their diagonal correlation function, as

$$
\begin{aligned}
C_{i j} & =0, \quad \text { for } \Gamma_{i j}>0 \\
& =0, \quad \text { for } \Gamma_{i j}<0,\left|\Gamma_{i j}\right|<\frac{1}{12} \\
& =6\left(\left|\Gamma_{i j}\right|-\frac{1}{12}\right), \quad \text { for } \Gamma_{i j}<0,\left|\Gamma_{i j}\right|>\frac{1}{12}
\end{aligned}
$$




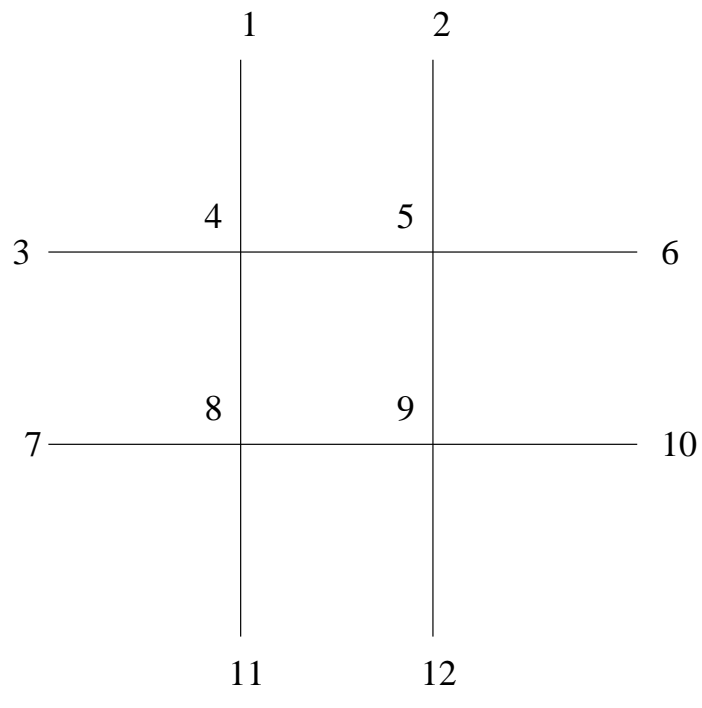

FIG. 1: A Tic-Tac-Toe cluster of twelve atoms. There are eight inequivalent pairs here. Only two inequivalent pairs of sites have nonzero concurrences in the ground state: $C_{1,2}=$ $0.08, C_{1,4}=0.41$.

The above formula substantially simplifies the procedure of calculating the pairwise concurrences in a many-qubit $S=0$ state. We simply calculate the diagonal spin-spin correlation function, and the concurrence is completely specified from the formula outlined. In the following section, we will effectively use the formula to study the entanglement distribution and sharing among various pairs in finite clusters and higher dimensional lattices.

Let us turn now to computing the actual values of the concurrences in one-dimensional systems. Now, for a closed chain of 12 sites, the above implies, denoting $C_{i j}=C_{r}$ where $r=j-i$, in the ground state $C_{1}=0.398$, and $C_{r}=0$ for $r>1$, where we have used the numerically computed diagonal correlation functions, $\Gamma_{1}=-0.1496, \Gamma_{2}=0.0626, \Gamma_{3}=-0.0553$ etc. . As the size of the chain is increased, the magnitude of the correlation function $\Gamma_{r}$ will take the limiting value from above. This would imply $C_{r}=0$, for $r>1$ for an infinite chain. $C_{1}$ can be estimated from the ground state energy in the thermodynamic limit. The ground state energy of the Heisenberg Hamiltonian in general can be written as

$$
E_{g} \equiv e_{g} N=3 N_{n} \Gamma_{1}
$$

where $e_{g}$ is the ground state energy per site, and $N_{n}$ the number of nearest-neighbor pairs of spins. Thus the nearest-neighbor concurrence can be written as

$$
C_{1}=6\left(\left|e_{g}\right| \frac{N}{3 N_{n}}-\frac{1}{12}\right)
$$

when positive, and otherwise zero. For a linear-chain antiferromagnet ground state, a similar formula has been derived [4]. The ground state energy is known from Bethe Ansatz, $e_{g}=\ln 2-1 / 4$, and $N_{n}=N$, which determines the nearest-neighbor concurrence, $C_{1}=2 \ln 2-1 \approx 0.386$.

\section{FINITE CLUSTERS AND EXTENDED TWO-DIMENSIONAL LATTICES}

In two-dimensional systems there are no analytical results for the ground state of Heisenberg antiferromagnet systems, like the Bethe-ansatz solution for the linear chain. Here, we will take recourse to numerical diagonalizations of finite clusters, and use variational estimates for the ground state energies on various extended lattices. The main difference between two-dimensional systems and the linear chain considered above is the number of nearest neighbors, apart from various local lattice structural differences. To gain insight into the entanglement distribution in two dimensions, we will numerically study below various finite clusters, which are drawn from square, triangular and Kagome' lattices.

Let us first consider a toy problem of a Tic-TacToe cluster of twelve sites, as shown in Fig.1. This cluster is drawn from a square lattice. Here there are two inquivalent sites, site 1 is equivalent to the other sites on the boundary, and site 4 is equivalent to the other sites forming the vertices of the inside square. There are eight inequivalent pairs of sites: $(1,2)$, $(1,3),(1,4),(1,5),(1,9),(1,10),(4,5),(4,9)$. From the numerical exact diagonalization for the $S=0$ ground state, only two inequivalent pairs have nonzero concurrence: $C_{1,2}=0.08, C_{1,4}=0.41$, and $C_{1,3}=C_{1,5}=C_{1,9}, C_{1,10}=$ $C_{4,5}=C_{4,9}=0$. In this finite cluster, the third-neighbor bonds, $(1,2),(1,6),(1,7),(1,11)$ have concurrence equal to 0.08 , where as the second-neighbor bonds $(1,5),(1,8),(1,3)$ etc. have zero concurrence. This can be ascribed to finite-size effects, and to the fact that this cluster does not have the same symmetry as the square lattice.

Let us now consider a 16-site square lattice cluster, with periodic boundary conditions. Here all sites are equivalent, with coordination number equal to four as on an infinite square lattice. This cluster has also the bipartite structure of the square lattice. There are only four inequivalent pairs, due to the periodic boundary conditions. For a given site, the second and third neighbors sit on the same sublattice, implying that the diagonal correlation function is positive, $\Gamma=0.071$ from the numerical calculation, and hence a zero concurrence. The numerical values of the correlation function are -0.117 and -0.067 for the nearest and furthest neighbor pairs respectively. Only the nearest-neighbor concurrence is nonzero, $C_{1}=0.202$ which is almost half the value we got for the linear chain. To study larger clusters, one has to increase the number of sites to 24 , to keep the bipartite structure intact. Then exact numerical diagonalization is quite difficult for such large clusters.

For the infinite square lattice the exact ground state energy is not known from analytical solutions, however, there are excellent estimates of upper bounds on $e_{g}$. For the square-lattice ground state[5], we have an upper bound estimate $\left|e_{g}\right| \approx 0.66$. This in turn, along with 


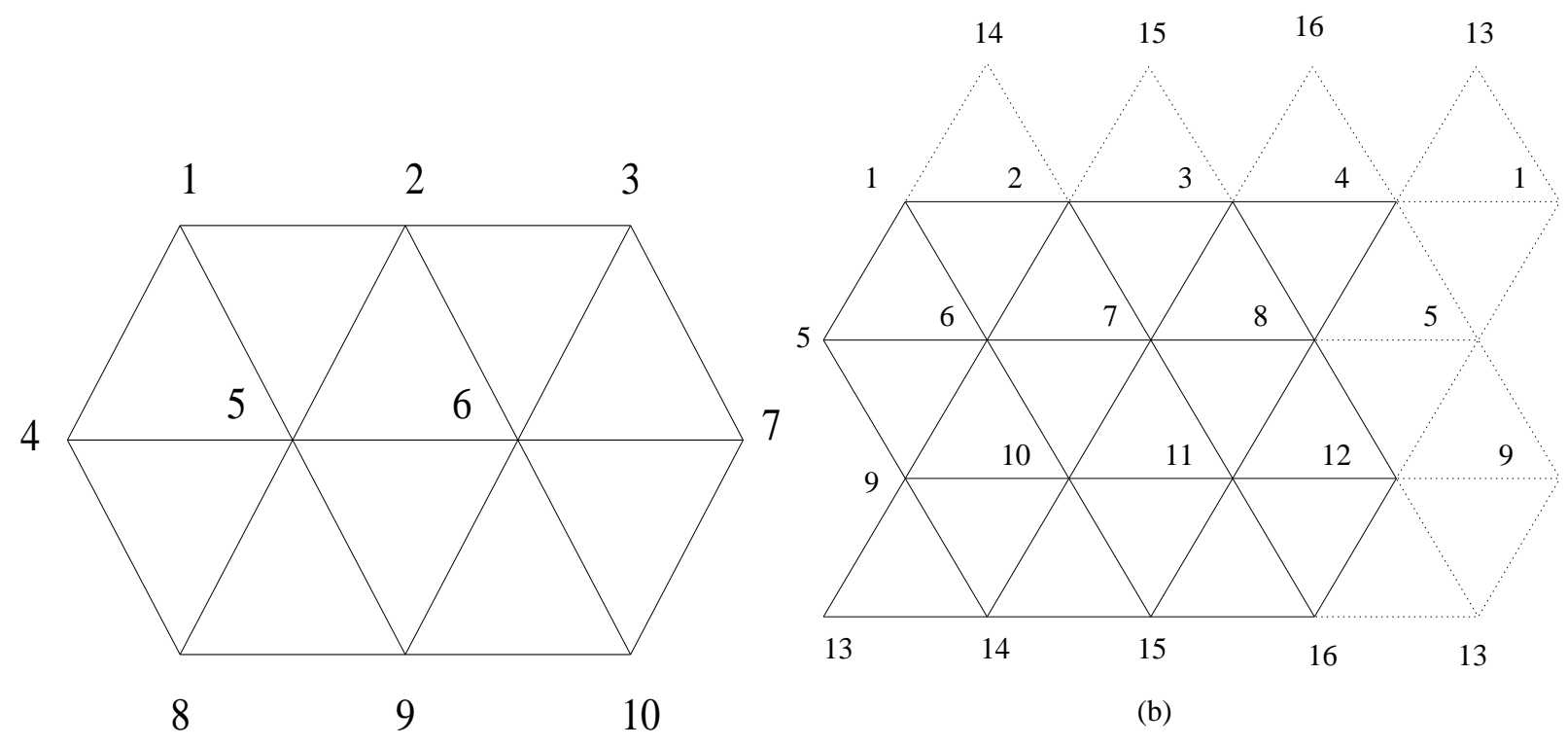

FIG. 2: (a) A triangular-lattice cluster with ten atoms. Here all the sites are not equivalent. There are four inequivalent sites labeled 1, 2, 4, 5. (b) A 16-site triangular cluster, with periodic boundary conditions. The extra nearest-neighbor bonds arising from the boundary conditions are shown with dotted lines. Here all sites are equivalent, with six nearest neighbors, like in the infinite triangular lattice. However, the horizontal bonds (sixteen in number) are not equivalent to the slanted bonds (32 in number).

$N_{n}=2 N$, implies

$$
C_{1}=\left|e_{g}\right|-\frac{1}{2} \approx 0.16 \text { for square lattice. }
$$

And the next-neighbor concurrence is zero, as the two spins sit on one sublattice of the square lattice. We conjecture that $C_{r}=0, r>1$ for square lattice also. This is because the concurrence reduces when the number of neighbors increases, as is evident from the nearestneighbor concurrence (it reduced from 0.386 to about 0.16 , as the number of nearest neighbors increased from 2 to 4 , from the linear chain to the square lattice). And in conjunction, we have the fourth-neighbor concurrence zero from the sixteen-site cluster studied above.

Let us now turn our attention to the triangular lattice, which has an increased number of nearest neighbors, along with a new ingredient of frustration among the spins on a triangular block, for minimization of energy. Again we take recourse to numerical diagonalization of finite clusters, in the absence of exact results for the ground state Heisenberg antiferromagnet. We will consider two different clusters here. Let us first look at a ten-site cluster shown in Fig.2a, which is drawn from a triangular lattice. Though all sites are not equivalent here, but it has the basic triangular and hexagonal block structure of a triangular lattice. From the numerical exact diagonalization for the ground state, only three inequivalent pairs have nonzero concurrence. The horizontal nearest-neighbor bond on the boundary has a concurrence, $C_{1,2}=0.26$, along with the three other equivalent bonds. The slanted nearest-neighbor bonds on the boundary have a concurrence, $C_{1,4}=0.347$. The bond at the center has a concurrence, $C_{5,6}=0.336$. All the other nearest-neighbor bonds have zero concurrence, along with all other pair concurrences between sites further apart. The magnitude of the nearest-neighbor pair concurrence has reduced here from the linear chain on account of the increased number of neighbors and the frustration associated with the triangular blocks.

Let us now consider a 16-site triangular cluster with periodic boundary conditions, shown in Fig.2b. The sites on the boundary get extra neighbors, due to the boundary conditions, indicated by dotted lines in the figure. For instance, site labeled as 1 has extra neighbors in sites labeled as 4,13 and 14, and so on for the other sites on the boundary (see figure). Now all the sites are equivalent, with six nearest neighbors as on an extended triangular lattice. This cluster has the ingredients of an infinite triangular lattice: six coordination, triangular block structure and associated frustration, equivalence of all the sites. One important ingredient that is missing here is the tripartite structure. There are two inequivalent nearest-neighbor bonds here, horizontal and slanted bonds. Starting from any site and traversing four horizontal bonds, one can return to the original site, whereas by traversing four slanted bonds one cannot return, as can be seen from the figure. From the numerical diagonalization, we get for the concurrence for the 16 horizontal bonds, $C_{1,2}=0$, and for the 32 slanted bonds, $C_{1,5}=0.05$. The concurrence is zero for all other pairs of sites further apart. Here, the concurrence is furthur reduced from that of the square-lattice clusters due to increased neighbors and frustration. This discrepancy between the horizontal bonds and the slanted bonds will 
disappear as the number of sites is increased, and the tripartite structure restored for an extended triangular lattice.

For an extended triangular lattice, we will use the variational estimates for the ground state energy, for calculation of nearest-neighbor concurrences. For a triangular lattice, we have $N_{n}=3 N$, which implies

$$
C_{1}=2\left(\frac{\left|e_{g}\right|}{3}-\frac{1}{4}\right) \text { for triangular lattice }
$$

if positive, and otherwise zero. The upper bound estimate for the ground state energy [5, [6] is $\left|e_{g}\right| \approx 0.53$. Hence, the nearest-neighbor concurrence for the triangular lattice is zero. The next-neighbor concurrence cannot be argued to be zero based on a bipartite structure, as we did above for the linear chain and the square lattice. However, we can argue that the absolute value of the correlation function would decrease as the separation increase. Also, from the 16-site cluster with six coordination discussed above, other than nearest-neighbor concurrences are vanishing. We expect as the size is increased, they will still be zero. This would imply $C_{r}=0$ for all separations on a triangular lattice.

We would like to study the Kagome' lattice clusters now. The obvious cluster that can be considered is a David star cluster of twelve sites shown in Fig.3, drawn from a Kagome' net. This cluster too has triangular blocks, along with the associated frustration, apart from unfilled hexagonal blocks unlike the triangular lattice. Numerically it is seen that this cluster has two degenerate $S=0$ ground states. The pair concurrences in one of the ground states, for the nearest-neighbor bonds can be calculated from the reduced density matrices. The only nonzero nearest-neighbor concurreces are for alternate bonds on the boundary, traversing clockwise, we have $C_{14}=C_{5,7}=C_{11,10}=C_{12,9}=C_{8,6}=C_{2,3}=0.55$. Similarly the other ground state has nonzero concurrences only for pairs $(1,3),(2,6),(8,9),(12,10),(11,7),(5,4)$. This is because these ground states have a large amplitude for singlets for these pairs. This can be seen easily by viewing the cluster shown in figure as a closed chain with every alternate site having a next-neighbor interaction. If every site has a next-neighbor interaction whose strength is half that of the nearest-neighbor interaction, then this reduces to Majumdar-Ghosh model, which has a two-fold degenerate valence-bond ground state 7]. But here, the next-neighbor interaction strength has been transfered to every second next-neighbor bond. But, still, the amplitude of the valence-bond configuration is predominant, giving rise to dominant contribution for concurrences only for alternate bonds. All the other pairs have zero concurrence. However, since the ground state is degenerate, one can change these concurrences by suitable linear combinations of the degenerate states. Larger clusters drawn from the Kagome' lattice, keeping the ingredients intact, are too large for exact diagonalization. Again, we will turn to the variational estimates for the ground state energy, to infer the nearest-neighbor

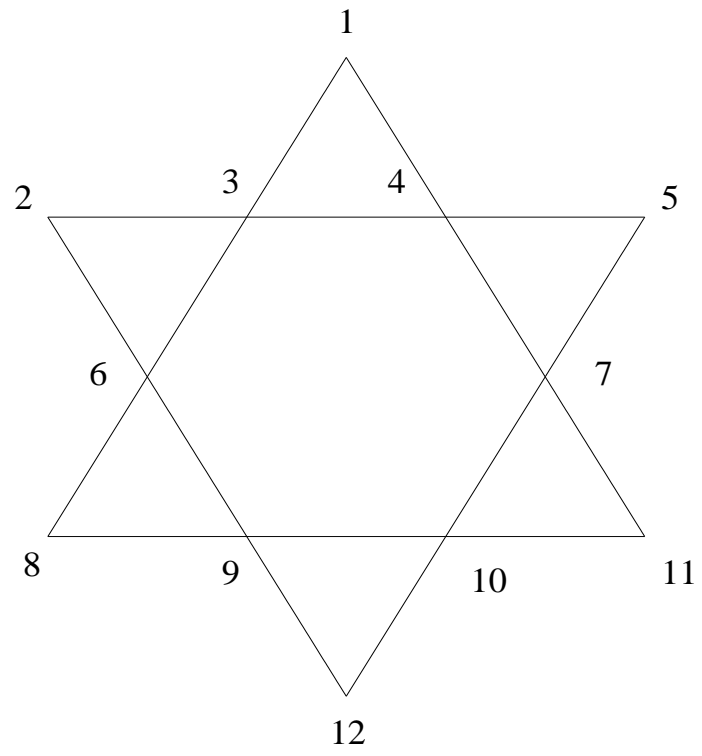

FIG. 3: A Kagome'-lattice David star cluster with twelve atoms. The ground state here is two-fold degenerate, with large amplitude for valance-bond configurations.

concurrences.

For the case of an extended Kagome' lattice, we have $N_{n}=2 N$, and the estimate for the ground state energy $\left[\underline{6},[],\left|e_{g}\right| \approx 5 / 12\right.$, would imply that the nearestneighbor concurrence is zero. Analogous to the triangular lattice, we can argue $C_{r}=0$ for all separations. Though the square lattice and the Kagome' lattice have the same number of nearest neighbors, $C_{1}$ is zero for Kagome lattice. This we can attribute to the frustration present in the Kagome' case, due to the presence of triangular blocks $[\underline{6}$.

We can increase the number of nearest neighbors by considering a $d$-dimensional hypercubic lattice, with $2 d$ nearest neighbors for every site. For large $d$, the ground state energy has an upper bound $\left|e_{g}\right| \approx d / 4$, and $N_{n}=$ $N d$ which will yield $C=0$ for the nearest neighbors. Similarly we expect all pairs of sites to have zero concurrence. This is consistent with our argument, that an increase in the number of neighbors will result in a reduction in the nearest-neighbor concurrences. We can also increase the effective number of neighbors by including a long-ranged interaction, by making the sum in the Heisenberg Hamiltonian (Eq.2) run over all pairs. Then each qubit interacts with all other qubits by the same strength. This gives us $H=\sum \vec{S}_{i} \cdot \vec{S}_{j}=\left(S^{2}-3 N / 4\right) / 2$, the eigenvalue depending only on the total spin quantum number $S$. A larger value for the total spin will lead to a larger energy. The lowest eigenvalue is for $S=0$, $e_{g}=-3 / 8$. Now, we also have $e_{g} N=3 N(N-1) \Gamma_{i j} / 2$, as all the two-point correlators would be identical, and $\vec{S}_{i} \cdot \vec{S}_{j}=3 \Gamma_{i j}$ for a $S=0$ state. This yields $C=0$, for all pairs of qubits. 


\section{MAXIMAL-SPIN STATES}

Let us turn our attention to the maximal-spin states, with $S=N / 2$, which are the ground states for a ferromagnetic interaction, and are maximal-energy states for the antiferromagnet, with an energy $E_{\max }=N / 4$. The structure of the maximal-spin states will be same for all clusters and lattices, irrespective of coordination number and local structure. There are $N+1$ such states, with one state each in a subspace with a given value $S^{z}=-N / 2, . . N / 2$. Let us write $S^{z}=N / 2-m$ where $m$ is the number of down spins in each basis state, $m=0,1 . . N$ for various subspaces. It is straightforward to write down the eigenstate for any lattice as

$$
\left|S=\frac{N}{2}, S^{z}=\frac{N}{2}-m>=\frac{1}{{ }^{N} C_{m}} \sum\right| i>
$$

where the sum is over ${ }^{N} C_{m}$ basis states each with $m$ down spins exactly. Since every basis state is given the same weight, the reduced density matrix for any pair of spins will have the same matrix elements, and the correlation functions, both the diagonal and the off-diagonal, will be same for every pair. This implies $C_{i j}=C$ for all the $N_{p}=N(N-1) / 2$ pairs. The diagonal correlation function, thus, can be written as $\Gamma_{i j}=$ $\left(1 / N_{p}\right) \sum_{\text {allpairs }}<S_{i}^{z} S_{j}^{z}>=\left(S^{z 2}-N / 4\right) / N(N-1)$. For values of $S^{z} \sim O(N)$, there is a long-ranged order. This would cause a decrease in the concurrence for a given pair, with a concurrence $\sim O(1 / N)$. However, the average concurrence for this case is more, as we will see below, than the concurrence in the $S=0$ state, that we discussed for various lattice systems above. Thus, the off-diagonal matrix elements of $R_{i j}$ are evaluated as

$$
\begin{aligned}
& u_{i j}=\frac{\left(\frac{N}{2}-S^{z}\right)\left(\frac{N}{2}-S^{z}-1\right)}{N(N-1)} \\
& v_{i j}=\frac{\left(\frac{N}{2}+S^{z}\right)\left(\frac{N}{2}+S^{z}-1\right)}{N(N-1)}
\end{aligned}
$$

Now, for the off-diagonal matrix element $z_{i j}=<$ $S_{j}^{+} S_{i}^{-}>$, the contributions come from all configurations with $\downarrow$ at site $j$ and $\uparrow$ at site $i$. There are $N-2 C_{m-2}$ such configurations. Hence,

$$
z_{i j}=\frac{\left(\frac{N}{2}-S^{z}\right)\left(\frac{N}{2}+S^{z}\right)}{N(N-1)} .
$$

Putting all things together, the concurrence for any pair of qubits is, with $S=N / 2, S^{z}=N / 2-m$,

$$
C(m)=\frac{2 m(N-m)}{N(N-1)}\left\{1-\sqrt{\frac{(m-1)(N-1-m)}{m(N-m)}}\right\} .
$$

A similar formula has been given in $[9]$. Some values of the function are $C\left(S=N / 2, S^{z}= \pm N / 2\right)=0$, and

$$
\begin{array}{r}
C\left(S=\frac{N}{2}, S^{z}=0\right)=\frac{1}{N-1} \\
C\left(S=\frac{N}{2},\left|S^{z}\right|=\frac{N}{2}-1\right)=\frac{2}{N} .
\end{array}
$$

The concurrence is maximum when the number of down spins is either 1 or N-1. Though all pairs have the same concurrence, however, the concurrence is not long ranged, since in the thermodynamic limit, $N \rightarrow \infty$, the concurrence goes to zero as $1 / N$. But, in comparison the state with $S=N / 2, S^{z}=0$ has a larger average concurrence than the state with $S=0=S^{z}$ that we studied earlier, where the best average concurrence for the linear chain is $\langle C>\approx 0.8 /(N-1)$. Thus, increasing the spin from $S=0$, for the ground state, to $S=N / 2$, for the maximal-energy state of the antiferromagnet, the nearest-neighbor concurrence has decreased from $O(1)$ to $O(1 / N)$. At the same time, the average over all pairs has improved, implying a better entanglement sharing in the maximal-spin states. Thus, the maximal-spin states with $S=N / 2,\left|S^{z}\right|<N / 2$ have a better entanglement sharing among the qubits, with the best sharing in the states $S=N / 2,\left|S^{z}\right|=N / 2-1$, with an average concurrence $<C>=2 / N$. The sectors with $S=N / 2-1, N / 2-2$, namely the one-magnon and two-magnon states, have been investigated [10, 11]. However, it remains to see if states with intermediate values of energy and/or with intermediate values of spin $0<S<N / 2-2$, can exhibit more average concurrence. Most important states are the first excited sates of the antiferromagnet, with $S=1$, where the concurrence results are yet to be known. It would be interesting to investigate the concurrence as a function of $S, S^{z}$ in all spin sectors.

\section{CONCLUSION}

We have investigated the quantum entanglement sharing in Heisenberg antiferromagnets using the concurrence measure. For $\mathrm{S}=0$ unique ground states, a simple formula relates the concurrence between an arbitrary pair of spins to their diagonal correlation function. This substantially simplifies the calculation of concurrences, both numerically and analytically. The nearest-neighbor concurrence is directly related to the ground state energy. For larger number of nearest-neighbors, the concurrence is smaller, as has been seen from studying a number of finite clusters, and from the study of large-d hypercubic lattice and longer-ranged interactions. It has been argued that other than nearest-neighbor pairs have zero concurrence in the ground state for the linear chain and the square lattice. It is shown that the nearest-neighbor concurrences also vanish in the ground state of triangular and Kagome lattice antiferromagnets. For maximal-spin states, explicit formulas are given for the pairwise concurrences, and the states with $S=N / 2, S^{z}=N / 2-1$ show maximum entanglement sharing. 
[1] Nielsen and Chuang, Quantum Computation

[2] W. K. Wootters, Phys. Rev. Lett. 80, 2245 (1998).

[3] Yu. A. Izyumov and Yu. N. Skryabin, Statistical Mechanics of Magnetically Ordered system, Consultants Bureau (New York, 1988)

[4] K. M. O'Connor and W. K. Wootters, Phys. Rev. A63, 052302 (2001); X. Wang and P. Zanardi, Phys. Lett. A301, 1 (2002).

[5] D. A. Huse and V. Elser, Phys. Rev. Lett. 60, 2531 (1988).
[6] V. Subrahmanyam, Phys. Rev. B52, 1133 (1995).

[7] C. K. Majumdar and D. P. Ghosh, J. Math. Phys. 10, 1388 (1969).

[8] V. Elser, Phys. Rev. Lett. 62, 3405 (1989).

[9] X. Wang and K. Moelmer, Eur. Phys. J. D18, 385 (2002).

[10] A. Lakshminarayan and V. Subrahmanyam, Phys. Rev. A67, 052304 (2003).

[11] V. Subrahmanyam and A. Lakshminarayan (Unpublished) 